\title{
Apolipoprotein B epitopes are present in nuclear preparations of human and mouse cells
}

\author{
KRISTINA DØSSING ${ }^{1}$ and SILVIO ZAINA ${ }^{1,2}$ \\ ${ }^{1}$ Department of Clinical Biochemistry, Rigshospitalet, Blegdamsvej 9, 2100 Copenhagen, Denmark; \\ ${ }^{2}$ Department of Medical Research, Division of Health Sciences, Campus Leon, \\ University of Guanajuato, 20 de Enero no. 929, 37000 Leon, Gto., Mexico
}

Received November 14, 2008; Accepted January 12, 2009

DOI: 10.3892/ijmm_00000202

\begin{abstract}
We previously showed that a native very lowdensity lipoprotein (VLDL)- and low-density lipoprotein (LDL)-rich mix induces global changes in DNA methylation in cultured THP-1 human macrophages. The exact molecular mechanisms for this response are not yet known. Previous studies showed that apolipoprotein B $(\mathrm{ApoB})$ or its fragments can be localized in nuclei following cellular uptake, thus suggesting that ApoB may have a chromatin-modifying activity. To verify this hypothesis, we assessed whether ApoB epitopes are detected in THP-1 and mouse cell nuclei. Using a combination of immunoblotting, immunocytochemistry and chromatin immunoprecipitation, we showed that ApoB epitopes are present in THP-1 cell and mouse monocyte/ macrophage nuclear fractions, but are perinuclear rather than intranuclear. Our results are not consistent with a direct chromatin-ApoB interaction as an underlying mechanism for the observed epigenetic responses to lipoproteins in THP-1 cells.
\end{abstract}

\section{Introduction}

We previously demonstrated that a mix of human native very low-, low- and high-density lipoproteins (VLDL, LDL and HDL, respectively) with a relatively high VLDL + LDL to HDL ratio induces global de novo DNA methylation, deacetylation of histone 4 and hypermethylation of lysine 20 of histone 4 (H4K20) in cultured THP-1 human macrophages (1). Furthermore, we demonstrated that LDL and VLDL were the most active lipoprotein species in inducing de novo DNA methylation and H4K20 hypermethylation (AlvaradoCaudillo et al, unpublished data). In the same study, we showed that fatty acids did not mimic any of the effects of

Correspondence to: Dr Silvio Zaina, Department of Medical Research, Division of Health Sciences, Campus Leon, University of Guanajuato, 20 de Enero no. 929, 37000 Leon, Gto., Mexico

E-mail: szaina@leon.ugto.mx

Key words: low-density lipoprotein, apolipoprotein B, macrophage, THP-1 cell line
VLDL and LDL on the epigenome, suggesting that these effects could be mediated by non-lipid lipoprotein components such as apolipoproteins. Among the latter, apolipoprotein B $(\mathrm{ApoB})$ is a potential candidate for at least two reasons. First, ApoB epitopes have been detected in the nuclei of rat liver cells (2). Second, in silico studies of ApoB polypeptide predicted nuclear localization sequence (NLS) and DNA binding domains (3). Perhaps the most intriguing observation supporting this hypothesis is the good efficiency of LDL as a DNA carrier in cell transfection experiments (3). These data point to a possible role for ApoB or its fragment(s) as a nuclear factor capable of affecting chromatin structure and gene expression or as a carrier of yet unidentified lipid species which perform this nuclear function (2). Here, we investigated whether ApoB localizes in the nucleus of THP-1 macrophages and directly interacts with chromatin.

\section{Materials and methods}

Lipoproteins and cell culture. Human VLDL, LDL and HDL were obtained and desalted as described (1). LDL was labeled with Texas Red as in the manufacturer's instructions (Molecular Probes, cat. no. T10244). THP-1 cell culture, differentiation and stimulation with human lipoproteins were performed as previously described (1). Caco-2 cells were cultured according to standard protocols. To isolate mouse monocytes and peritoneal macrophages, anaesthetized animals were bled by decapitation. Monocytes were isolated from anticoagulated blood by gradient in Ficoll-Paque PLUS (Amersham Biosciences). To isolate peritoneal macrophages, cells obtained by peritoneal wash with RPMI-1640 medium were allowed to adhere to plastic overnight and were then collected by scraping.

Nuclei isolation and analysis. Nuclear preparations were obtained essentially as described elsewhere (4) with the following modifications. Following cell disruption with $10 \%$ NP-40 as described in the original procedure, nuclear material was further purified by centrifugation through a $10 \%(\mathrm{w} / \mathrm{v})$ sucrose cushion and resuspended in $0.25 \mathrm{M}$ sucrose and $0.35 \%$ Triton $\mathrm{X}-100$.

For immunoblot analysis, nuclei were resuspended thoroughly in complete SDS-PAGE buffer. The following anti-human ApoB antibodies were used: Dako A/S cat. no. 

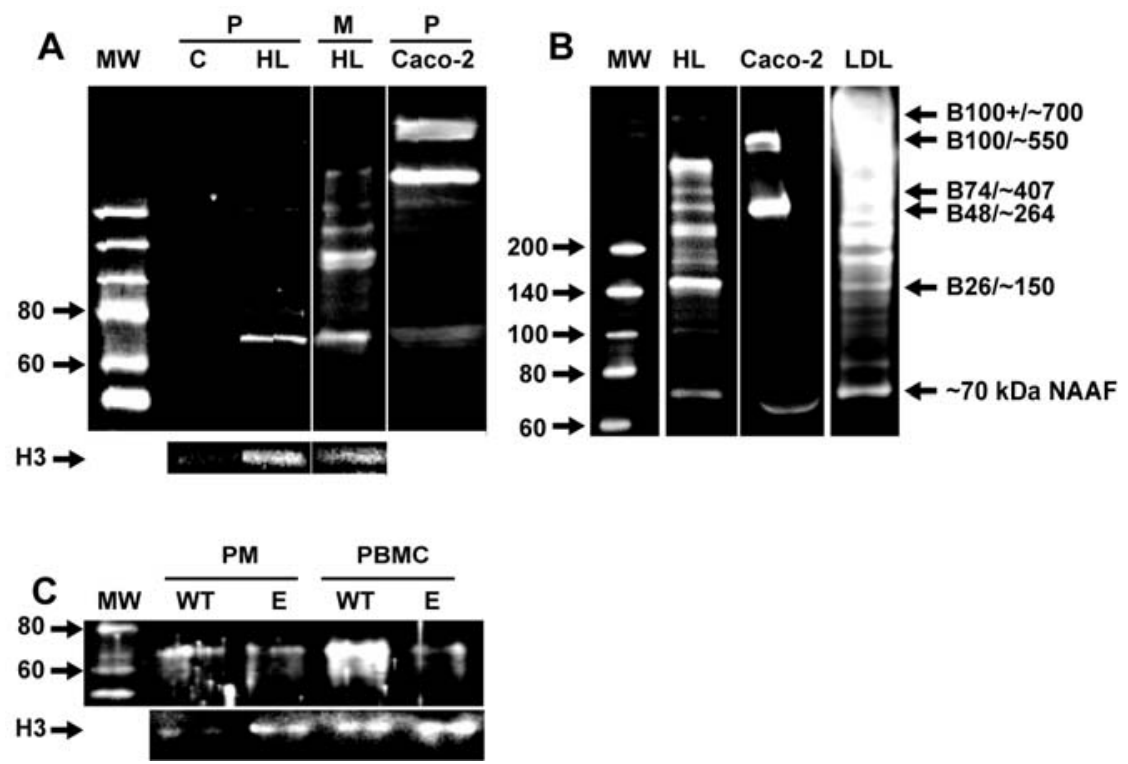

Figure 1. ApoB epitopes in human and mouse cell nuclear preparations. (A) Nuclei from control and HL-stimulated THP-1 macrophages probed with a polyclonal anti-ApoB antibody (Dako A/S) or with the 1D1 monoclonal antibody ( $\mathrm{P}$ and $\mathrm{M}$, respectively). A representative banding pattern from Caco-2 cell medium is shown as a positive control for antibody specificity. C and HL indicate controls (unstimulated) and hyperlipidaemic lipoprotein mix, respectively. $\mathrm{H} 3$ is a histone 3 loading control. MW standards with corresponding sizes (in kDa) are shown on the left. (B) High MW resolution of ApoB epitopes present in $500 \mu \mathrm{g} / \mathrm{ml}$ LDL-stimulated THP-1 macrophage nuclear preparations probed with the 1D1 antibody. The identity of ApoB species is indicated on the right. (C) Presence of an $\sim 70 \mathrm{kDa}$ ApoB epitope in peritoneal macrophage (PM) and peripheral blood mononuclear cell (PBMC) nuclear preparations from WT and ApoE-deficient (E) mice probed with a polyclonal antibody (BioSite). Symbols as in panel A.

Q0497, Abcam cat. no. ab8947 (polyclonals), 1D1 and MB24 (monoclonals, kind gifts from Lars Bo Nielsen, Department of Clinical Biochemistry, Rigshospitalet, Denmark). The antimouse ApoB was from BioSite (cat. no. K23300R). AntiApoA-I and -ApoE antibodies were from Rockland cat. no. 600-101-196 and Abcam cat. no. ab7620, respectively. Antihistone $\mathrm{H} 3$ antibodies (Upstate cat. no. 05-499 or 06-755) were used for loading controls. Anti-LAMP1, 2 and 3 and anti-Golgin 97 (Santa Cruz Biotechnology cat. nos. sc-8098, sc-8100, sc-15363 and Abcam cat. no. ab15845, respectively) antibodies were used to detect lysosomal and Golgi markers.

The lysosomal enzyme assays were carried out in freshly isolated nuclei according to Ludwig et al (5) using 4methylumbelliferyl- $\beta$-D-galactopyranoside (Sigma cat. no. M1633) as glucuronidase-specific substrate.

Immunocytochemistry. Cells were fixed for $15 \mathrm{~min}$ in fresh PFA (4\%), washed in PBS, permeabilized for 5 min with PBS and $0.1 \%$ Triton X-100 and blocked for $1 \mathrm{~h}$ with PBS and $2 \% \mathrm{BSA}$ at room temperature, before being probed with anti-nucleolin (polyclonal, Abcam cat. no. ab22758) or antihistone H3 (monoclonal, Upstate Biotechnology cat. no. 05499) antibody overnight in PBS and $2 \%$ BSA at $4^{\circ} \mathrm{C}$. After primary antibody incubation, cells were washed in PBS and incubated with secondary antibody for a minimum of $1 \mathrm{~h}$ in PBS and $2 \%$ BSA at $4{ }^{\circ} \mathrm{C}$ and subjected to three 5-min washes in PBS. Specimens were examined on a Zeiss LSM 510 confocal laser-scanning microscope using a x40 objective.

Chromatin immunoprecipitation (ChIP). ChIP was performed essentially as described (6) with Dako A/S cat. no. Q0497 anti-ApoB antibody $(1: 1,000)$ and salmon sperm DNA/ protein A-agarose (Upstate cat. no. 16-157) in TBS.
Immunoprecipitated DNA was cloned in Zero Blunt ${ }^{\circledR}$ TOPO $^{\circledR}$ (Invitrogen cat. no. K287540).

\section{Results}

ApoB fragments are present in human and mouse cell nuclear preparations. To assess whether ApoB epitopes localize to the nucleus of THP-1 macrophages, nuclear preparations were probed with anti-ApoB antibodies by Western blotting. THP-1 macrophages were stimulated with a lipoprotein mix resembling plasma lipoprotein levels of hyperlipidaemic ApoE-null mice, i.e. $68.8 \mu \mathrm{g} / \mathrm{ml}$ VLDL, $32.1 \mu \mathrm{g} / \mathrm{ml} \mathrm{LDL}$, $91.1 \mu \mathrm{g} / \mathrm{ml} \mathrm{HDL}$ (referred to as hyperlipidaemic lipoprotein mix or HL; all concentrations are in $\mu \mathrm{g}$ protein $/ \mathrm{ml}$ ) for $24 \mathrm{~h}$. All nuclear fractions were free of lysosomal and Golgi material as judged from the absence of LAMP1, 2 and 3 and Golgin 97 (assessed by Western blotting) and glucuronidase (assessed by direct enzyme assay, data not shown).

Western blot analysis revealed that two polyclonal (Dako and Abcam) and two monoclonal (1D1 and MB24) antiApoB antibodies consistently detected a predominant putative ApoB epitope of $\sim 70 \mathrm{kDa}$ in nuclear preparations of THP-1 macrophages stimulated with the HL mix, but not in unstimulated cells (Fig. 1A; for simplicity only Dako antibody results are shown). In addition, MB24 and 1D1 monoclonal antibodies detected at least 5 high MW epitopes (Fig. 1A; only 1D1 antibody results are shown). Furthermore, ApoB-secreting Caco-2 cell medium probed with polyclonal anti-ApoB antibodies yielded ApoB100 and 48 epitopes, suggesting that the observed nuclei-associated ApoB fragments (NAAFs) were not unspecifically recognized polypeptides (Fig. 1A). Notably, Caco-2 cell media consistently showed an additional $\sim 70-\mathrm{kDa}$ fragment similar to the one observed 

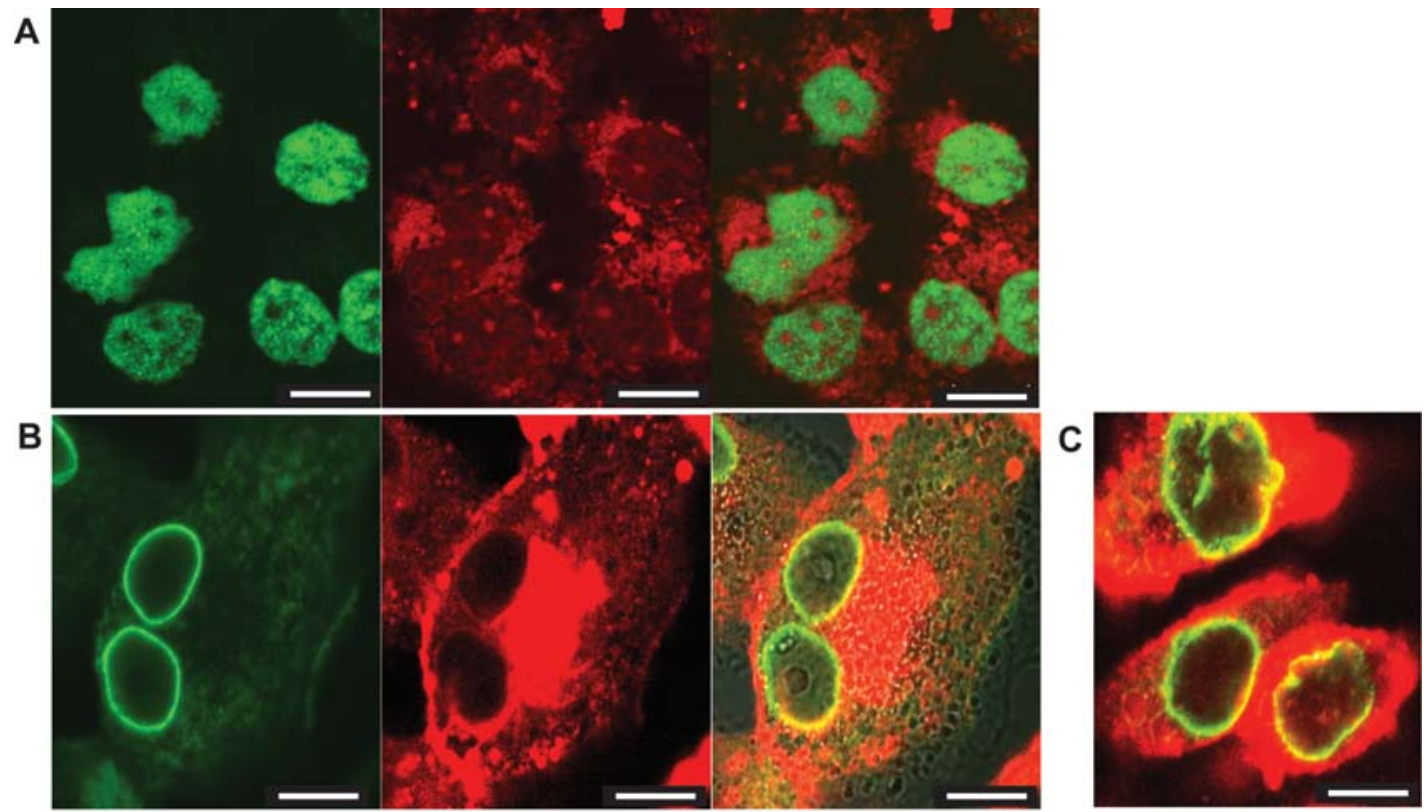

Figure 2. Perinuclear localization of ApoB. Immunocytochemistry images of THP-1 macrophages stained by incubation with $100 \mu \mathrm{g} / \mathrm{ml}$ Texas Red-labeled LDL for $24 \mathrm{~h}$ and further probed with an anti-histone H3 (A) or anti-nuclear pore complex (NPC) (B and C) antibody. A and B represent green (NPC or histones), red (Texas Red), and combined fluorescence images. (C) A further example of combined green/red fluorescence images. White bar, $10 \mu \mathrm{m}$.

in THP-1 macrophages. Furthermore, human LDL itself contains several low MW ApoB epitopes including the $\sim 70$-kDa band, suggesting that this NAAF is present in lipoproteins prior to internalization in THP-1 macrophages, rather than being a product of intracellular processing (Fig. 1A). ApoB epitopes recognized by the monoclonal antibodies used in this study, i.e. 1D1 and MB24, have been well characterized and allow us to tentatively assign the nuclear 70-kDa epitope to the amino terminal portion of ApoB (7). In addition, the observed ApoB epitopes are in good agreement with previously identified ApoB species. When high MW proteins were allowed to resolve in SDS-PAGE we observed a fragment resembling by size ApoB $100^{+}$, an $\mathrm{ApoB}$ species believed to be an oligomer of ApoB100 and/or the $144-\mathrm{kDa}$ ApoB26 weighing $\sim 700 \mathrm{kDa}(8)$. Furthermore, we detected the 407-kDa ApoB74, ApoB26 and the 70-kDa ApoB fragment (2,7-9) (Fig. 1B).

Furthermore, in order to rule out that the predominant $\sim 70 \mathrm{kDa}$ NAAF is unique to the THP-1 cell line, we probed nuclear preparations from ApoE-deficient and wild-type (WT) mouse peritoneal macrophages and peripheral blood mononuclear cells (PBMCs) with a mouse polyclonal antiApoB antibody. Noticeably, a predominant ApoB epitope of $\sim 70 \mathrm{kDa}$ was also detected by this polyclonal antibody in mouse cells and consistently at higher levels in WT compared to ApoE-deficient mouse cell nuclei (Fig. 1C). The observation that the $\sim 70-\mathrm{kDa}$ NAAF is detected in ApoEdeficient mice is genetic proof that this NAAF does not result from antibody cross-reaction with ApoE. In addition, we ruled out cross-reaction with Apo-AI (see below) and BSA (data not shown) by performing specific immunoblotting.

$N A A F s$ are perinuclear. The nuclear localization of ApoB was further studied by immunocytochemistry in THP-1 macrophages incubated with $100 \mu \mathrm{g} / \mathrm{ml}$ Texas Red-labeled LDL for various times. ApoB strongly accumulated within the cytoplasm in a time-dependent fashion and showed an additional apparently nuclear localization following a 24-h incubation (Fig. 2A). Nonetheless, the latter was likely to represent a fixation artifact, as judged by overlapping with nuclear pore complex-labeled material (Fig. 2B and C) and lack of any colocalization with histones (Fig. 2A).

In order to independently validate the results obtained by immunocytochemistry, we performed chromatin immunoprecipitation (ChIP) with polyclonal anti-ApoB antibodies. The degree of chromatin-ApoB interaction was assessed by counting colonies generated by transformation with plasmids containing DNA fragments immunoprecipitated from nuclei of HL mix-stimulated THP-1 cells. Two independent ChIPs performed with anti-ApoB antibodies yielded an average of 6 colonies per plate (cpp). Anti-ApoB ChIP efficiency was extremely low in comparison with a positive control ChIP performed under the same conditions with an anti-acetylated histone 3 antibody which yielded an average of $83 \mathrm{cpp}$. A negative control ChIP performed omitting the anti-ApoB antibody generated an average of $2 \mathrm{cpp}$. Anti-ApoB ChIP yielded repetitive sequences belonging to Alu ( 8 clones) and IAP ( 3 clones), in addition to one human aorta EST (GenBank D79585).

Prompted by these findings, we performed an in silico analysis of ApoB to identify putative domains interacting with perinuclear or nuclear membrane factors. We submitted the full-length $H$. sapiens ApoB polypeptide (accession no. AAP72970) to a conserved domain search using NCBI/ BLAST. The analysis predicted a putative SbcC domain $\left(\mathrm{E}=9 \times 10^{-5}\right)$ between positions $2100-2600$, conserved in M. musculus and R. norvegicus. SbcC is a bacterial ATPase involved in DNA repair, replication and recombination and 


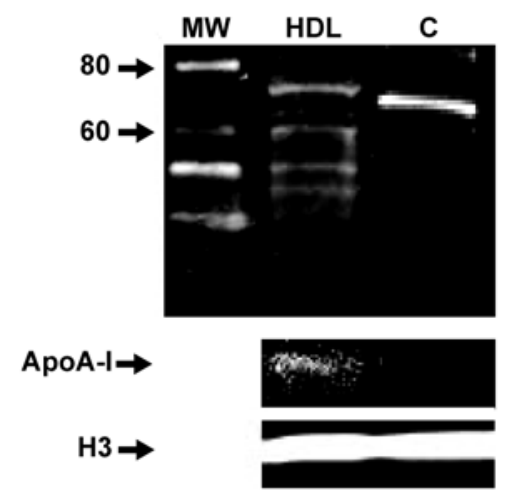

Figure 3. HDL modifies the NAAF pattern. Nuclear preparations from HLstimulated THP-1 macrophages were further stimulated with $100 \mu \mathrm{g} / \mathrm{ml}$ HDL or buffer (HDL and C, respectively) for $24 \mathrm{~h}$ and probed with the 1D1 monoclonal anti-ApoB antibody. Note the presence of the ApoA-I in HDLstimulated cell nuclei. Symbols as in Fig. 1 legend.

belongs to a subclass of SMC (Structural maintenance of chromosomes) proteins $(10,11)$. The same analysis did not yield any SbcC domain in ApoE or ApoA-I, nor did it reveal the previously identified nuclear localization signal (4). The latter discrepancy likely reflects differences in the prediction method.

$H D L$ alters the pattern of NAAF. If the perinuclear localization of ApoB fragments is pathobiologically relevant, it is expected that the atheroprotective HDL would alter NAAF levels and/or composition (12). We verified this hypothesis by subjecting THP-1 macrophages to a further $24-\mathrm{h}$ stimulation with $100 \mu \mathrm{g} / \mathrm{ml}$ HDL after HL mix stimulation. The analysis revealed a markedly changed banding pattern, i.e. a much fainter band intensity and an increased number of bands compared to the corresponding pattern observed in nuclei from cells stimulated with HL alone (Fig. 3). The experiment allowed us to rule out that the detected ApoB epitopes resulted from cross-reaction with ApoA-I, as the latter was detected as a 30-kDa band as expected (Fig. 3).

\section{Discussion}

Our study showed that monoclonal and polyclonal antibodies detect ApoB fragments in nuclear preparations of human HL and LDL-stimulated THP-1 cells, mouse PBMCs and peritoneal macrophages. These fragments consist mainly of a $\sim 70-\mathrm{kDa}$ band present in both species and additional bands of apparent MW between ApoB100 and 70 kDa. The observation that the $\sim 70-\mathrm{kDa}$ NAAF is more abundant in WT than ApoEdeficient cell nuclei is interesting but counter-intuitive and deserves further study. Furthermore, we demonstrated by immunocytochemistry that the bulk of nuclei-associated ApoB is perinuclear, while ChIP analysis indicated that NAAFs are not in physical contact with chromatin. Similarly, our in silico analysis of ApoB did not predict any DNA binding domain or nuclear localization signal as detected in a previous study (3). The significance of the predicted bacterial SbcC domain is uncertain and needs to be supported by further thorough analysis.
Our results are in general agreement with previous data supporting a nuclear proximity and a cytoplasm-to-nucleus shuttle function for ApoB in liver cells $(2,3)$. Nonetheless, we could not unequivocally establish an intranuclear localization for ApoB in the cell types examined, suggesting that cell type-specific differences in ApoB localization may exist. Yet, perinuclear localization does not exclude a nuclear transport activity for ApoB, perhaps in concert with nucleolin, since the latter was shown to specifically bind ApoB- and ApoEcontaining lipoproteins and to shuttle between the nucleus and the cytoplasm (13). At any rate, our results do not support our original hypothesis that intracellular lipoprotein processing produces ApoB fragments that mediate the observed effects of lipoproteins on DNA methylation by direct interaction with chromatin (14). Furthermore, our recent data demonstrate that lipoprotein fatty acids induce changes in DNA methylation and histone modifications that are distinct from the ones induced by intact lipoprotein particles. Taken together, these observations imply that lipoproteins contain yet unknown modifiers of the epigenome.

One question posed by our findings involves the origin of the observed nuclei-associated ApoB fragments. We suggest that these are contained in lipoproteins rather than being a product of intracellular lipoprotein processing, since we detected ApoB epitopes migrating below ApoB100 in human LDL. These findings are supported by the observation that ApoB-synthesizing liver HepG2 cells show ApoB fragments of similar size and patterns as observed in our experiments (15). Albeit apparently not consistent with accepted knowledge of LDL composition, the latter result is unlikely to be biased by failing antibody selectivity, since Caco- 2 cell culture medium shows a clear ApoB100 and B48 doublet, indicating that our anti-ApoB antibodies adequately detect the major ApoB forms. In addition, Caco- 2 cell culture medium consistently showed a weak ApoB epitope apparently comigrating with the $\sim 70-\mathrm{kDa} \mathrm{NAAF}$, indicating that at least the latter NAAF could be incorporated in nascent lipoproteins.

One further crucial issue involves the pathophysiological relevance of nuclei-associated ApoB fragments. We observed that HDL alters the distribution of such fragments, thus possibly reflecting the profound functional differences between HDL and ApoB-rich lipoproteins in the atherogenic process (12). Whether and how these responses relate to the observed differences in epigenetic effects between HDL-rich and -poor lipoprotein mixes and among individual lipoproteins cannot be concluded at present (1). Notably, we detected ApoA-I, a major HDL constituent, in nuclear preparations of HDL-stimulated cells in accordance with previous reports suggesting that HDL-derived factors may well operate as nuclear factors $(2,16)$. This idea is supported by recent evidence that the HDL atypical apolipoprotein ApoD can be detected within the nuclear space $(17,18)$. Further experimental work will be necessary to confirm the pathobiological relevance of these findings.

\section{Acknowledgements}

We gratefully acknowledge Kristian Klindt and Stine Østergaard for the technical assistance, Marie Wickström Lindholm for supplying native lipoproteins and THP-1 cells and for 
discussions relevant for these experiments, and Emil D. Bartels for kindly providing the Caco-2 cell media. This work was supported by Hjerteforeningen (Danish Heart Association), Lundbeck Foundation and Novo Nordisk Foundation.

\section{References}

1. Lund G, Andersson L, Lauria M, Lindholm MW, Fraga MF Villar-Garea A, Ballestar E, Esteller M and Zaina S: DNA methylation polymorphisms precede any histological sign of atherosclerosis in mice lacking Apolipoprotein E. J Biol Chem 279: 29147-29154, 2004.

2. Panin LE, Russkikh GS and Polyakov LM: Detection of apolipoprotein A-I, B and E immunoreactivity in the nuclei of various rat tissue cells. Biochemistry 65: 1684-1689, 2000

3. Guevara JG Jr, Kang D and Moore JP: Nucleic acid binding properties of low-density lipoproteins: LDL as a natural gene vector. J Protein Chem 18: 845-857, 1999.

4. Schreiber E, Matthias P, Müller MM and Schaffner W: Rapid detection of octamer binding proteins with 'mini-extracts', prepared from a small number of cells. Nucleic Acids Res 17: 6419,1989

5. Ludwig T, Ovitt CE, Bauer U, Hollinshead M, Remmler J, Lobel P, Rüther U and Hoflack B: Targeted disruption of the mouse cation-dependent mannose 6-phosphate receptor results in partial missorting of multiple lysosomal enzymes. EMBO J 12: 5225-5235, 1993.

6. Su RC, Brown KE, Saaber S, Fisher AG, Merkenschlager M and Smale ST: Dynamic assembly of silent chromatin during thymocyte maturation. Nat Genet 36: 502-506, 2004.

7. Pease RJ, Milne RW, Jessup WK, Law A, Provost P, Fruchart J-C, Dean RT, Marcel YL and Scott J: Use of bacterial expression cloning to localize the epitopes for a series of monoclonal antibodies against apolipoprotein B100. J Biol Chem 265: 553-568, 1990.

8. Curtiss LK and Edgington TS: Immunochemical heterogeneity of human plasma apolipoprotein B I. Apolipoprotein B binding of mouse hybridoma antibodies. J Biol Chem 257: 15213-15221, 1982.
9. Milne RW, Blanchette L, Théolis R Jr, Weech PK and Marcel YL: Monoclonal antibodies distinguish between lipiddependent and reversible conformational states of human apolipoprotein B. Mol Immunol 24: 435-447, 1987.

10. Connelly JC, Kirkham LA and Leach DRF: The SbcCD nuclease of Escheria coli is a structural maintenance of chromosomes (SMC) family protein that cleaves hairpin DNA. Proc Natl Acad Sci USA 95: 7969-7974, 1998.

11. Löwe J, Cordell SC and van den Ent F: Crystal structure of the SMC head domain: An ABC ATPase with 900 residues antiparallel coiled-coil insert. J Mol Biol 306: 25-35, 2001.

12. Kannel WB, Castelli WP and Gordon T: Cholesterol in the prediction of atherosclerotic disease. New perspectives based on the Framingham study. Ann Intern Med 90: 85-91, 1979.

13. Semenkovich CF, Ostlund RE Jr, Olson MO and Yang JW: A protein partially expressed on the surface of HepG2 cells that binds lipoproteins specifically is nucleolin. Biochemistry 29: 9708-9713, 1990.

14. Zaina S, Døssing KBV, Lindholm MW and Lund G: Chromatin modification by lipids and lipoprotein components: an initiating event in atherogenesis? Curr Opin Lipidol 16: 549-553, 2005

15. Liao W, Strube RW, Milne RW, Chen S-Y and Chan L: Cloning of ApoB intrabodies: specific knockdown of ApoB in HepG2 cells. Biochem Biophys Res Commun 373: 235-240, 2008.

16. Panin LE, Tuzikov FV and Gimautdinova OI: Tetrahydrocortisol-apolipoprotein A-I complex specifically interacts with eukaryotic DNA and GCC elements of genes. J Steroid Biochem Mol Biol 87: 309-318, 2003.

17. Bouma M-E, de Bandt JP, Ayrault-Jarrier M, Burdin J, Verthier N and Raisonnier A: Immunoperoxidase localization of apolipoprotein D in human enterocytes and hepatocytes. Scand J Gastroenterol 33: 477-483, 1988.

18. Do Carmo S, Levros L-C Jr and Rassart E: Modulation of apolipoprotein $\mathrm{D}$ expression and translocation under specific stress conditions. Biochim Biophys Acta 6: 954-969, 2007. 